University of Wollongong

Research Online

Faculty of Engineering and Information

Faculty of Engineering and Information

Sciences - Papers: Part A

Sciences

2016

\title{
Effects of hydraulic pressure on wrinkling and earing in micro hydro deep drawing of SUS304 circular cups
}

\author{
Liang Luo \\ University of Wollongong, II895@uowmail.edu.au \\ Dongbin Wei \\ University of Wollongong, dwei@uow.edu.au \\ Xiaogang Wang \\ Taiyuan University of Science and Technology \\ Cunlong Zhou \\ Taiyuan University of Science and Technology, zcunlong@163.com \\ Qingxue Huang \\ Taiyuan University of Science and Technology
}

See next page for additional authors

Follow this and additional works at: https://ro.uow.edu.au/eispapers

Part of the Engineering Commons, and the Science and Technology Studies Commons

Research Online is the open access institutional repository for the University of Wollongong. For further information contact the UOW Library: research-pubs@uow.edu.au 


\title{
Effects of hydraulic pressure on wrinkling and earing in micro hydro deep drawing of SUS304 circular cups
}

\begin{abstract}
Influences of hydraulic pressure on forming features in micro hydro deep drawing are different from those in normal drawing due to the small size of specimens. In this study, micro hydro deep drawing of SUS304 sheets was carried out in order to study the impacts of the hydraulic pressure on the quality of the drawn cup. Experimental results indicate that there is a critical hydraulic pressure range from 3 to $6 \%$ of the blank's initial yield stress, where wrinkling and earing development trends change twice. The wrinkling and the earing of the drawn cup also reach their local extremes in the critical pressure range. The cup earing value moves in the opposite direction from the wrinkling value. Hydraulic pressure affects the wrinkling and the earing of the drawn cup through changes in the micro-frictional condition, the shape of the blank and its strain-stress state. Micro-finite element (FE) simulation which takes these factors as well as the material size effects into consideration showed similar results to the experimental ones, thus validating the experimental results and the suitability of the micro-simulation model for micro-forming FE simulation. The experimental and simulation results indicate that the critical hydraulic pressure based on the blank's initial yield stress can restrict the wrinkling and the earing of the drawn cup. Ultra-high pressure has the potential to avoid the cup wrinkling and earing.
\end{abstract}

\section{Keywords}

circular, sus304, drawing, deep, hydro, cups, micro, effects, earing, wrinkling, pressure, hydraulic

Disciplines

Engineering | Science and Technology Studies

\section{Publication Details}

Luo, L., Wei, D., Wang, X., Zhou, C., Huang, Q., Xu, J., Wu, D. \& Jiang, Z. (2017). Effects of hydraulic pressure on wrinkling and earing in micro hydro deep drawing of SUS304 circular cups. The International Journal of Advanced Manufacturing Technology, 90 189-197.

\section{Authors}

Liang Luo, Dongbin Wei, Xiaogang Wang, Cunlong Zhou, Qingxue Huang, Jianzhong Xu, Di Wu, and Zhengyi Jiang 
Effects of hydraulic pressure on wrinkling and earing in micro hydro deep drawing of SUS304 circular cups

\author{
Liang Luo, Zhengyi Jiang ${ }^{*}$, Dongbin Wei, Zhengyi Jiang ${ }^{\dagger}$ \\ University of Wollongong, Australia \\ Xiaogang Wang, Cunlong Zhou, Qingxue Huang, Taiyuan University of Science and \\ Technology, China \\ Jianzhong Xu, Di Wu, State Key Laboratory of Rolling and Automation, Northeastern \\ University, China
}

\begin{abstract}
Influences of hydraulic pressure on forming feature of cups are different in micro hydro deep drawing due to size effects. In this study, the micro hydro deep drawing of SUS304 sheets was conducted to study the impacts of the hydraulic pressure on drawn cups' quality. Experimental results indicate that there is a critical hydraulic pressure range from three to six percent of blank's initial yield stress, where wrinkling and earing developing trends change twice. The wrinkling and earing also reach their local extremums in the critical pressure range. The earing is strongly related to the wrinkling phenomenon. Friction plays an important role in micro hydro deep drawing and significantly affects the wrinkling and the earing of the drawn cup. Moreover, micro scale simulation with consideration of the size effects and friction change presents similar wrinkling development trend to that in the experiments, which proves the influence and importance of the size effects and friction change.
\end{abstract}

Keywords: Micro hydro deep drawing, Size effects, Wrinkling, Earing, Micro simulation

\title{
1 Introduction
}

Hydraulic pressure is a key factor in hydro forming processes. It has been used for improvement of drawability, simplification of processing steps and promotion of product's quality [1-4]. In traditional hydro forming processes, for example, hydro deep drawing, the hydraulic pressure is generally corresponding with the drawing process $[5,6]$. A low hydraulic pressure for the prebulging followed by an increasing pressure at the first half and decreasing pressure at the second half of the drawing process offers good drawn products. Moreover, a proper pre-bulging process enhances the materials' formability due to reverse loading and work hardening [7]. During the drawing process, the hydraulic pressure reduces disadvantageous friction between a blank and a die, while increases beneficial contact between the blank and a punch, which acts as a holding force. Furthermore, wrinkling is limited due to increase of contact force between the blank and a

\footnotetext{
*Corresponding author: jiang@uow.edu.at

${ }^{\dagger}$ Corresponding author: jiang@uow.edu.au
} 
blank holder at the cost of enlarged adverse friction force. Additionally, effective fillet radii of the blank near the punch nose and die shoulder reduced with the hydraulic pressure. Therefore, moderate pressure keeps a balance between shape accuracy and material drawability due to the reduction of the effective fillet radii. Overall, the hydraulic pressure improves the forming process through the change of the material strain-stress state, friction conditions and transitional geometry during the forming process.

Driven by increasing requirements of micro metal products, micro deep drawing has been introduced to manufacture various micro cups and boxes. With an attempt to improve the quality of micro deep drawn cups, micro hydro deep drawing (MHDD) has been introduced [8-11]. However, the size effects change the impacts of the hydraulic pressure in the micro hydro deep drawing [12]. The change of material properties directly influences deformation of the blank [13]. Rated geometrical parameters, such as the bending ratio at the die corner and punch nose and relative punch diameter, are different due to the thickness of the blank [14, 15]. Thus, the drawing process is impacted. Furthermore, the friction condition in micro scale is different from that of normal scale drawing process. Correspondingly, the open and closed lubricant pockets theory has been used as an explanation of the friction change [16, 17]. Moreover, these changes affect fluid development which influences sheets' deformation as a consequence. Regarding the fluid, the leakage, flow state and boundaries effects are all different in tiny gaps between the blank and the die [18]. Moreover, the interaction of the deformation of the blank and the development of fluid further increases the complexity of the influences of the hydraulic pressure in the micro hydro deep drawing.

In this study, the micro hydro deep drawing experiments were conducted to investigate the impacts of the hydraulic pressure. The drawn cups were observed under a digital microscope and analysed by an image processing program. Consequently, the influence of the hydraulic pressure on the wrinkling and earing of the drawn cup was discussed. Finally, micro scale finite element simulation considering the size effects and the micro scale friction was run and compared with experiments.

\section{Experimental}

\subsection{Material}

SUS304 sheets with a thickness of $50 \mu \mathrm{m}$ were divided into three groups and annealed at $975{ }^{\circ} \mathrm{C}$, $1050{ }^{\circ} \mathrm{C}$ and $1100{ }^{\circ} \mathrm{C}$ for two minutes in an argon gas protection atmosphere respectively. Thus their microstructures were of equiaxial crystals and average grain sizes were 10, 20 and $40 \mu \mathrm{m}$ correspondingly. With the change of the microstructures, mechanical properties, such as initial yield strength and ultimate tensile strength, were altered. Fig. 1 shows (a) the microstructures of the three SUS304 sheets groups, and (b) their true strain-stress curves from tensile tests results. 
The micro hydro deep drawing system was developed based on a micro deep drawing system. Due to tiny sizes of the raw material, a one-stroke blanking-drawing process was applied for the micro deep drawing system. Fig. 2(a) displays the sketch of the one-stroke blanking-drawing system. During the first half stroke, a blanking die and a blanking holder moved downwards together while the die was fixed. The die acted as a blanking punch and the blank for the subsequent micro deep drawing was prepared. In addition, the hydraulic pressure was not supplied at this stage. At the end of the first half stroke, the blank holder moved to its limiting position and held the blank with the die via a constant gap during the second half stroke. Next, the pressured oil was applied. Simultaneously, the punch continued drawing down and drew a cup under the hydraulic pressure. Therefore, the whole MHDD process was completed and the cup was produced. Because of this design, difficulties in positioning and transporting of raw material were solved.

(a)

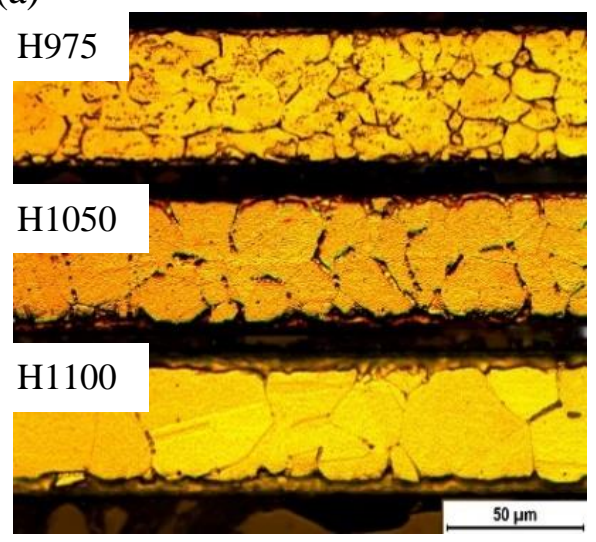

(b)

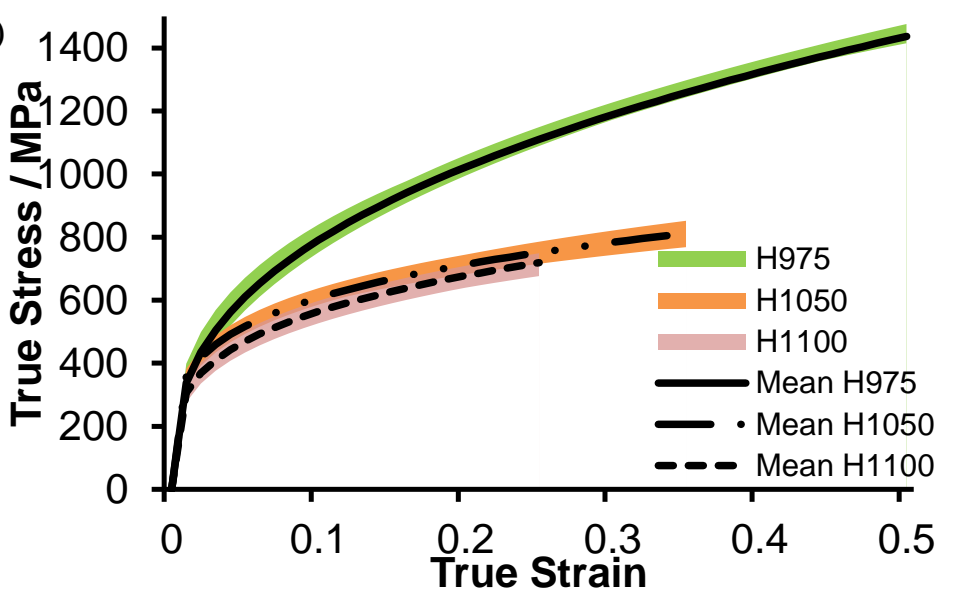

Figure 1 (a) Microstructures of the three sheets groups and (b) their tensile tests results 
(a)

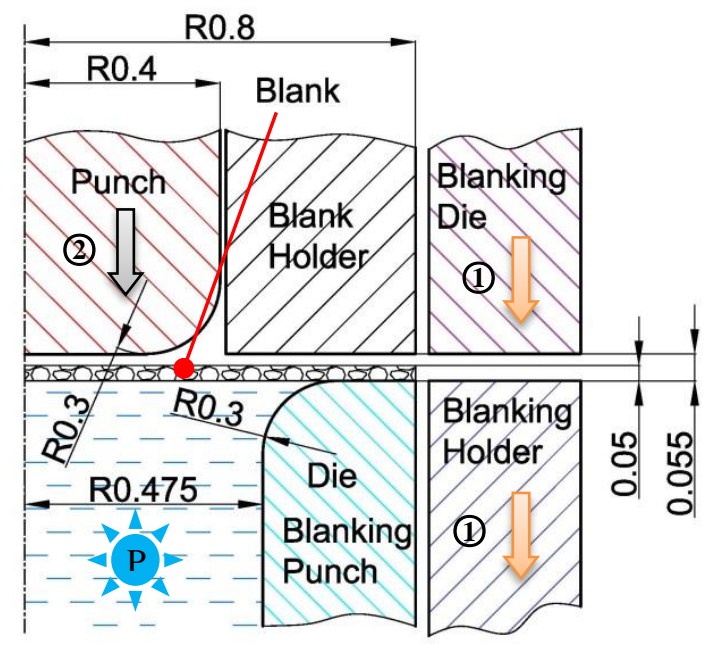

(b)

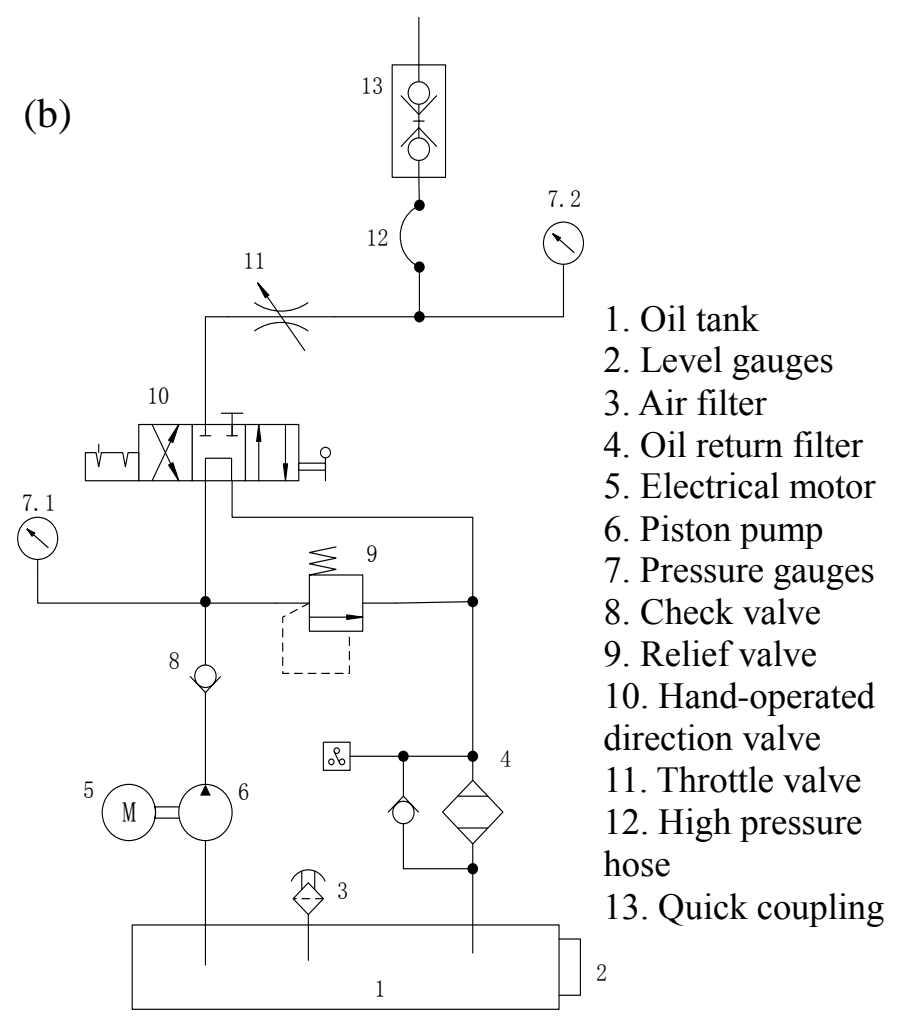

Figure 2 Sketches of (a) the MHDD and (b) hydraulic systems

A hydraulic station was linked to the micro deep drawing system to supply the hydraulic pressure. Its systematic view is shown in Fig. 2(b). Mechanical hydraulic oil with a viscosity of 46 cSt was utilised as working media. The handle-operated directional valve (\#10) was of a three-position four-path valve with the ' $M$ ' function. At the start of the experiments, the valve was at its middle functional position. Therefore, the oil could flow back to the oil tank (\#1) through the handle-operated valve without loading. When preparing for the working pressure, the handle-operated valve was moved to its left functional positon and the oil supplied by the pump (\#6) was blocked. Until the hydraulic pressure reached the designed working pressure which is controlled by the relive valve (\#9), the oil flowed back to the oil tank through the relive valve. Once all settings were ready, the handle-operated valve was set to its right functional position and thus the pressed oil was supplied for the MHDD experiments. Once completed one experiment, the hydraulic station can be separated from the micro deep drawing system through the quick coupling (\#13) without stopping the pump. Moreover, there is no need for resetting the working pressure for a next experiment. One of the pressure gauges (\#7.1) was used for pressure setting while the other one (\#7.2) was utilised for monitoring the hydraulic pressure during the MHDD experiments. Furthermore, the throttle valve (\#11) was employed for adjustment of the oil flow rate. Because of the minute dimensions of the micro deep drawing system, there is no space for sealing parts. Therefore, a tiny gap between the die/blank holder and the blanking die/blanking holder was used to seal the pressured oil. As the gaps were about $2 \sim 4 \mu \mathrm{m}$, the oil leakage was small and the pressure lost was limited. By contrast, the deformation of the blank compressed the fluid field and increased the hydraulic pressure. However, compared to the 
relatively large initial fluid volume, the pressure increase was minute and ignorable. Thus, the relatively stable hydraulic pressure can be maintained during the MHDD experiments.

\subsection{Experimental conditions}

The surface of all sheets were slightly polished via a soft eraser and then cleaned by alcohol before the MHDD experiments. Therefore, contamination on the surface of the sheet was removed. The drawing speed was $0.1 \mathrm{~mm} / \mathrm{s}$ and the gap between the blank holder and the die was fixed as $55 \mu \mathrm{m}$ according to the thickness of the sheets. Moreover, the hydraulic pressure was only added at the drawing process. To research the influences of the hydraulic pressure, all three groups of sheets were drawn under a serial of hydraulic pressures. Furthermore, the hydraulic pressure was increased from $5 \mathrm{MPa}$ to $30 \mathrm{MPa}$ at an incremental step of $5 \mathrm{MPa}$. After the drawing process, the drawn cup was removed from the mouth of the die. Then, the cups were treated by an ultrasonic cleaner for one minute within the alcohol cleaning media before observation under a high-resolution laser-assisted digital microscope (VK-X1000/X2000 series from (OKEYENCE). Repeated experiments were conducted for each experimental condition and each material group.

\section{Results and discussion}

As the wrinkling is a normal defect of a drawn cup, the wrinkling phenomenon was firstly studied. Accordingly, the drawn cup's mouth was observed under the microscope and measured by a developed image processing program in MATLAB. A 3D image was firstly generated from a serial of images at sequential forcing heights from the laser-assisted microscope. Therefore, the cup was coloured based on height information. Next, both inner and outer boundaries of the cup's mouth were detected by the developed image processing program based on the colour of the images. Moreover, the colourful digital image was firstly transferred into binary image and only cup's mouth was marked as white. Next, its inner and outer boundaries were detected by the Moore-Neighbour tracing algorithm modified by Jacob's stopping criteria in MATLAB. Consequently, the minimum circumscribed circle (MCC) and the maximum inscribed circle (MIC) of each boundary were fitted separately by MATLAB. Fig. 4(a) shows a drawn cup, and (b) displays the detected boundaries and their fitted circles. The diameter difference between the maximum and minimum fitted circles was chosen as a judgement of the wrinkling, as shown in Eq. (1). Fig. 5 displays the wrinkles of both the outer and inner surface of different drawn cups under the designed hydraulic pressures. Due to the size effects, normalised pressure, the ratio of the hydraulic pressure to the initial yield stress, was utilised for analysis. 


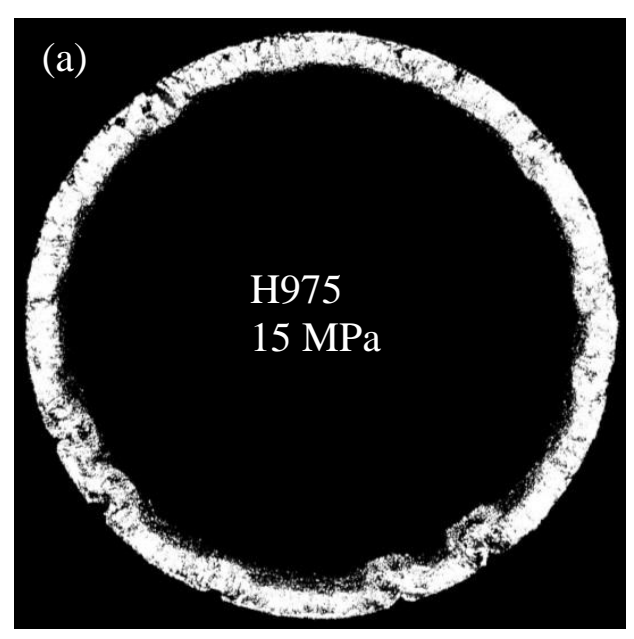

(b)

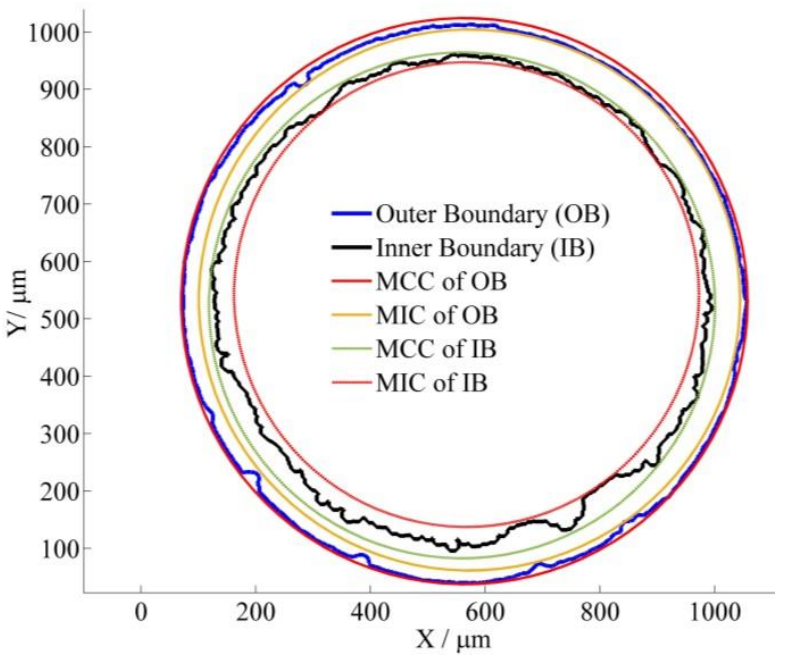

Figure 4 (a) Drawn cup and (b) its detected boundaries with fitted circles

$$
W^{i}=\text { max diameter }{ }^{i}-\text { min diameter }{ }^{i}
$$

where $W$ is the wrinkling factor and the subscript $i$ indicates the inner or outer boundary.

The wrinkling development was complicated with the hydraulic pressure for all material groups. Under low hydraulic pressure, the wrinkling values increased with the hydraulic pressure. Once reached a critical value, the wrinkles dropped to a local minimum. Thereafter, the wrinkles rose with the hydraulic pressure till the maximum experimental pressure. Moreover, the inner wrinkles were greater than the outer wrinkles for each material group under each hydraulic pressure.

The wrinkling occurs when energy consumption on the primary deformation path is higher than that on the secondary deformation path [19]. The wrinkling (secondary) deformation energy consumption contains bending work on each wrinkle and thickness direction blank holding work, as shown in Eqs. (2) and (3) respectively. Moreover, the primary deformation energy is circumferential compression work, as shown in Eq. (4). 
(a)
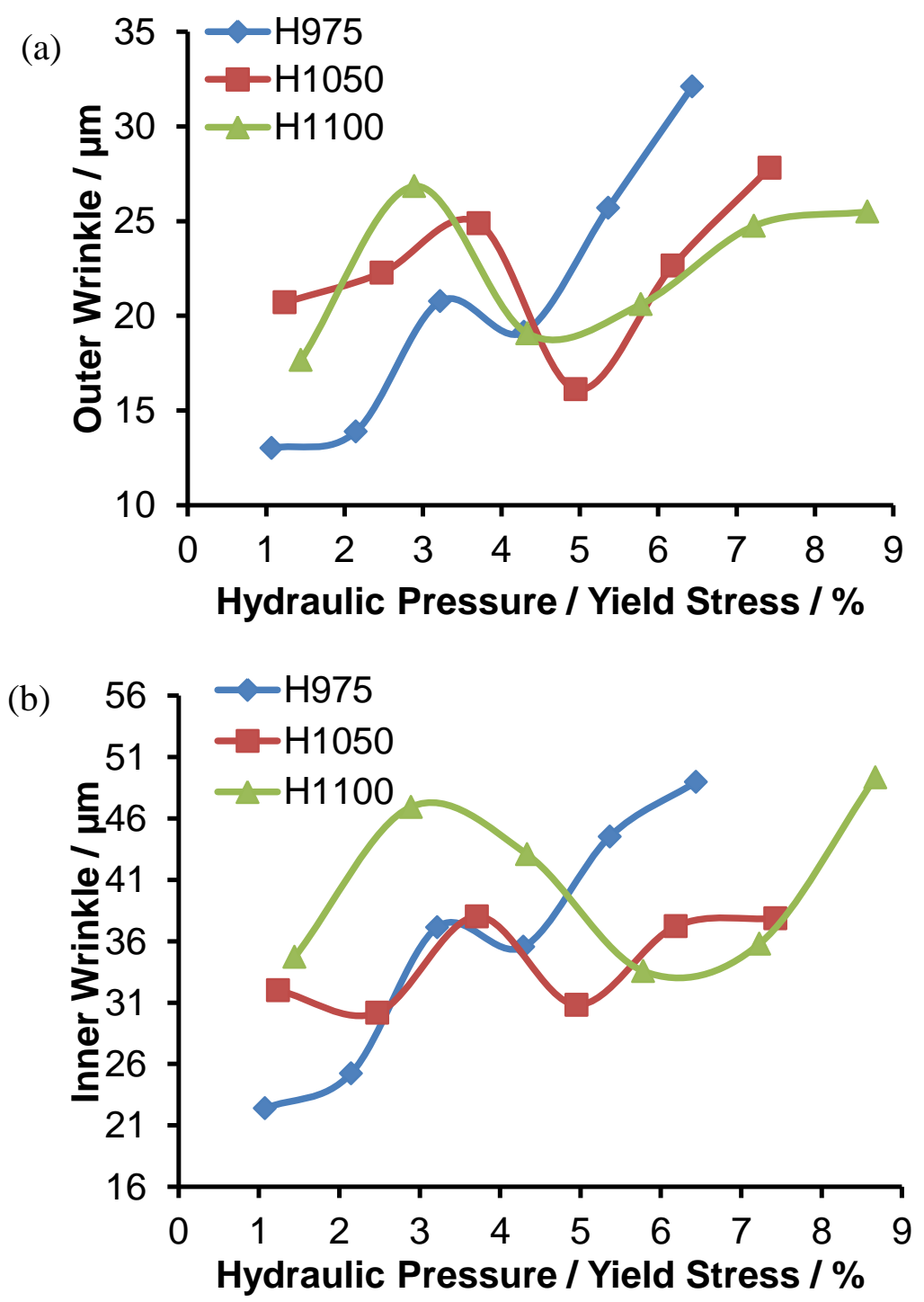

Figure 5 Wrinkles of the (a) outer and (b) inner surfaces of drawn cups

$$
\begin{gathered}
E_{b}=\frac{E_{r} I}{2} \int_{0}^{l}\left(\frac{d^{2} y}{d x^{2}}\right)^{2} d x \\
E_{q}=\int_{0}^{l} \int_{0}^{y} b q d y d x \\
E_{\theta}=\bar{\sigma}_{\theta} b t \times \frac{1}{2} \int_{0}^{l}\left(\frac{d^{2} y}{d x^{2}}\right)^{2} d x
\end{gathered}
$$

where $E_{b}, E_{q}$ and $E_{\theta}$ are the bending work, the thickness direction blank holding work and the compression work correspondingly. $E_{r}$ is the equivalent modulus, given by Eq. (5), $I$ is the inertia moment, $l$ is the length of a single wrinkle, $\bar{\sigma}_{\theta}$ is the compression stress, $b$ is the width of the wrinkle, $t$ is the thickness, and $q$ is the effective blank holding force, $x$ and $\mathrm{y}$ are the circumferential length and height of the wrinkle. 


$$
E_{r}=\frac{4 E D}{(\sqrt{E}+\sqrt{D})^{2}}
$$

where $E$ is the elastic modulus and $D$ is the slope of strain-stress curve at the special stress state in plastic deformation period. According to the bifurcation theory, wrinkling condition can be expressed by Eq. (6).

$$
E_{\theta}>E_{b}+E_{q}
$$

At the edge of the blank where is potential wrinkling area, the force balance condition of a slice of blank is illustrated by Fig. 6 (a). The radial and thickness direction force balance equations can be presented by Eqs. (7) and (8) respectively. Initially, the blank contacted with the blank holder. However, as the blank was drawn into the die, the blank holder did not press the blank due to the geometry of the MHDD system as shown in Fig. 6(b). Moreover, the compression stress increased and wrinkles occurred since the blank was departed from the blank holder. Thus, the effective blank holding force, $q$, can be expressed as Eq. (11).

(a)

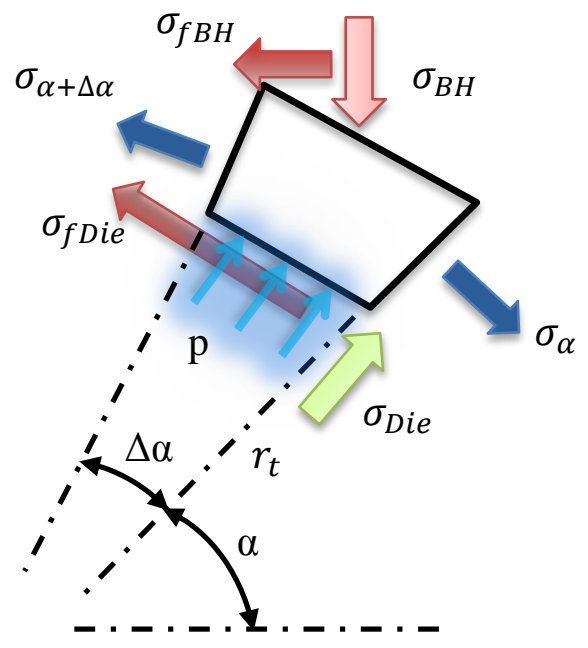

(b)

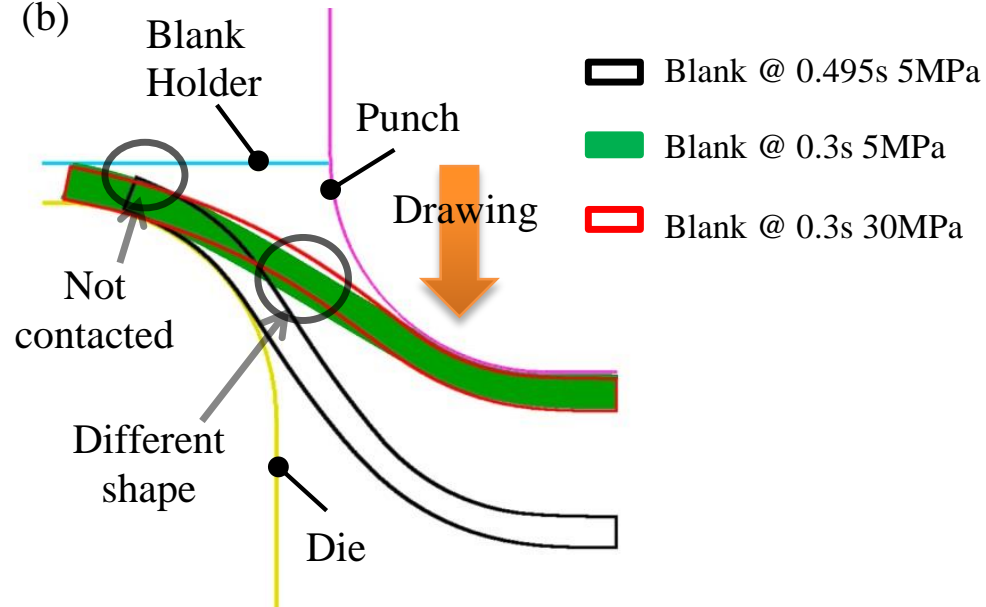

Figure 6 Illustrations of (a) stress state at the edge of the blank and (b) blank's geometry during MHDD

$$
\begin{gathered}
P+\sigma_{\text {Die }}=\sigma_{B H} \cdot \sin \alpha+\sigma_{f B H} \cdot \cos \alpha+\sigma_{f D i e}+\sigma_{\alpha+\Delta \alpha} \cdot \sin \Delta \alpha \\
\sigma_{\alpha+\Delta \alpha} \cdot \cos \Delta \alpha+\sigma_{f D i e}+\sigma_{f B H} \cdot \sin \alpha=\sigma_{\alpha}+\sigma_{B H} \cdot \cos \alpha \\
\sigma_{f D i e}=\sigma_{D i e} \cdot \mu(P) \\
\sigma_{f B H}=\sigma_{B H} \cdot \mu^{\prime} \\
q= \begin{cases}\sigma_{B H} \cdot \sin \alpha+\sigma_{\alpha+\Delta \alpha} \cdot \sin \Delta \alpha & \text { (Initial) } \\
\sigma_{\alpha+\Delta \alpha} \cdot \sin \Delta \alpha & \text { (Later) }\end{cases}
\end{gathered}
$$

where $P$ is the hydraulic pressure, $\sigma_{D i e}$ is the support from the die, $\sigma_{\alpha}$ and $\sigma_{\alpha+\Delta \alpha}$ are the average radial tensile stresses on thickness section at the angles of $\alpha$ and $\alpha+\Delta \alpha$ respectively, $\sigma_{f D i e}$ and $\sigma_{f B H}$ are the friction forces from the die and the blank holder on unit area correspondingly, as 
shown in Eqs. (9) and (10) respectively, $\sigma_{B H}$ is the blank holding force on unit area, and $\mu(P)$ is the frictional coefficient which is related to the hydraulic pressure, while $\mu^{\prime}$ is the frictional coefficient between the blank and the blank holder.

At low pressure conditions, the hydraulic pressure did not change the shape of the blank significantly. However, frictional force reduced due to the increase of lubricant flow rate and pressure. Consequently, the radial force, $\sigma_{\alpha+\Delta \alpha}$, decreased, resulting in small effective blank holding force as shown in Eq. (11). According to the wrinkling criteria, Eq. (6), the possibility of wrinkling increased with the hydraulic pressure at low pressure conditions.

With further increase of the hydraulic pressure, the shape of the blank during MHDD was influenced significantly, as shown in Fig. 6(b). Actual radius of the blank near the die shoulder was reduced leading to the small angle of radius, $\alpha$, and small bending curvature. Consequently, the radial tensile stress and effective blank holding force increased. As a result, the wrinkling potential reduced.

By contrast, at high hydraulic pressure conditions, the high pressure decreased the effective blank radius near the die shoulder as well as the friction between the blank and the die. Moreover, the stress state was changed to a degree that the compression stress decreased with the hydraulic pressure, as shown in Eq. (12). Overall effects of all wrinkling factors resulted in an increase of wrinkles.

$$
\sigma_{\theta}^{\prime}=\bar{\sigma}+\bar{\sigma}_{\theta}
$$

where $\bar{\sigma}$ is the hydro static stress and $\sigma^{\prime}{ }_{\theta}$ is the overall compression stress.

The critical pressure for each material group was in a range between three and six percent of the initial yield stress. In addition, according to Eq. (7), the blank contacted with the die more tightly than that with the punch. Therefore, the inner surface of the blank had looser limitation and greater wrinkles than the outer surface. Additionally, due to the size effects, the material flow stress was reduced, resulting in small equivalent modulus, $E_{r}$. Therefore, the overall wrinkling in the MHDD was higher than that in the normal scale hydro deep drawing. As grain number on the thickness direction was small, the plastic deformation mechanism was different. As a result, the relationship of the wrinkling values for all material groups under each hydraulic pressure was different. Furthermore, due to the material inhomogeneity, wrinkles were randomly distributed along the edge of the drawn cup, as shown in Fig. 4(a).

The earing in the normal deep drawing process is mainly because of material anisotropy. However, the friction force plays an important role and influences the earing in the micro hydro deep drawing. The height information along a circle on the drawn cup's mouth from the 3D image was selected and its average waviness height difference, as shown in Eq. (13), was utilised as an earing judgement. Waviness presents a surface that appears periodically at longer intervals than the roughness [20]. Thus, through a cut-off filter, which is a low pass Gaussian filter, the roughness information was transferred into earing information. Fig. 7 displays (a) the 3D drawn cup and (b) the earing of each experimental case. 


$$
\text { Earing }=\frac{1}{n} \sum_{i=i}^{n}\left|w_{i}-\bar{w}\right|
$$

where $w_{i}$ is the wave height, $\bar{w}$ is the average wave height of all $n$ waves.

(a)

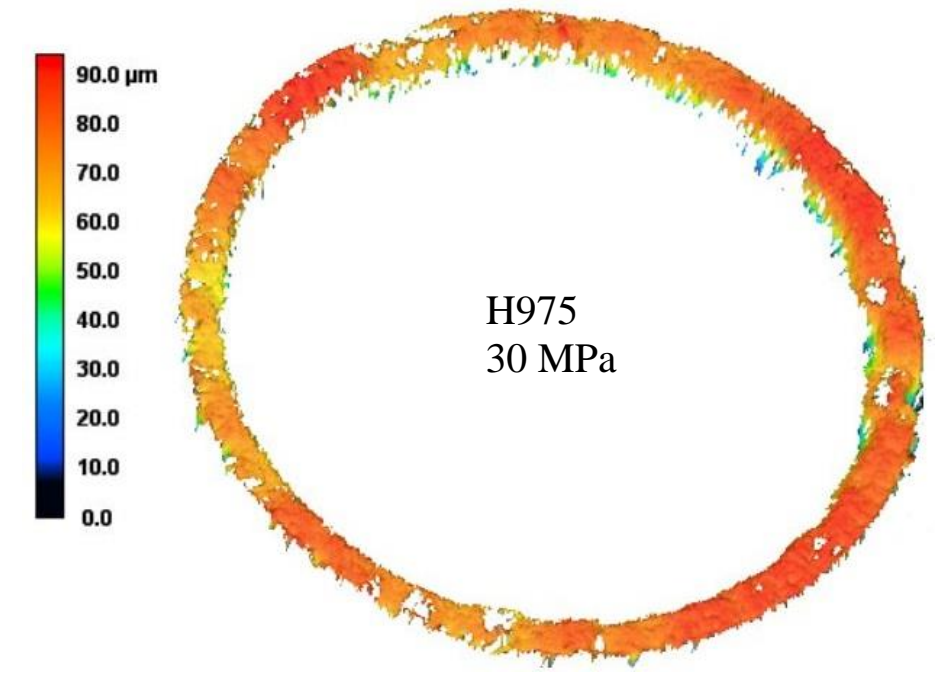

(b)

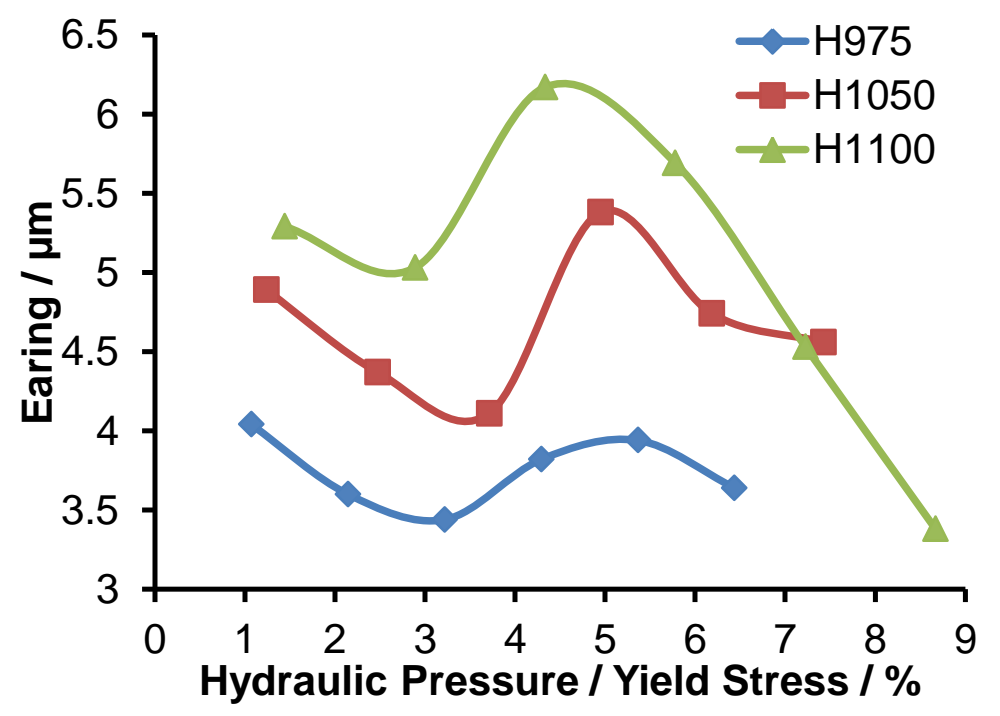

Figure 7 (a) The 3D drawn cup and (b) the earing of each experimental condition

The general earing developing trend was reverse to the wrinkling tendency. The earing values for each material group decreased firstly and then rose to a local peak. Since the critical pressure, the earing reduced continually. Moreover, the earing increased with the annealing temperature, while the earing of $1100^{\circ} \mathrm{C}$ heated material was the smallest at the $30 \mathrm{MPa}$ pressure experimental condition.

At low pressure, the oil lubrication effects on the drawing process were not significant and material inhomogeneity played an important role in the earing phenomenon. With an increase of the hydraulic pressure, serious wrinkling occurred and changed frictional condition, as shown in Fig. 8. Due to the wrinkling, the friction between the blank and the punch was increased considerably. Moreover, the wrinkled peak that contacted with the punch bore a downward 
friction force, whereas the bottom of the wrinkle shouldered an upward friction force from the die. The friction forces on both sides of the blank were in opposite directions and acted on different places. Further, the wrinkles height affected the contact force and consequently the friction force. Thus, the earing reduced with the friction transformation. Once reached the critical hydraulic pressure, the wrinkling was dropt, and therefore the friction transformation was weakened, resulting in an increase of earing. By further increasing the hydraulic pressure, friction transformation was recovered with the increase of the wrinkling. Moreover, much more effective oil lubrication was built, and consequently the friction between the blank and the die was released, while the friction between the blank and the punch was enhanced. Therefore, the earing was further decreased at high hydraulic pressures. Furthermore, under the high pressure, wrinkling values and wrinkled area was increased significantly in the $1100^{\circ} \mathrm{C}$ treated material group, leading to great limitation of earing.
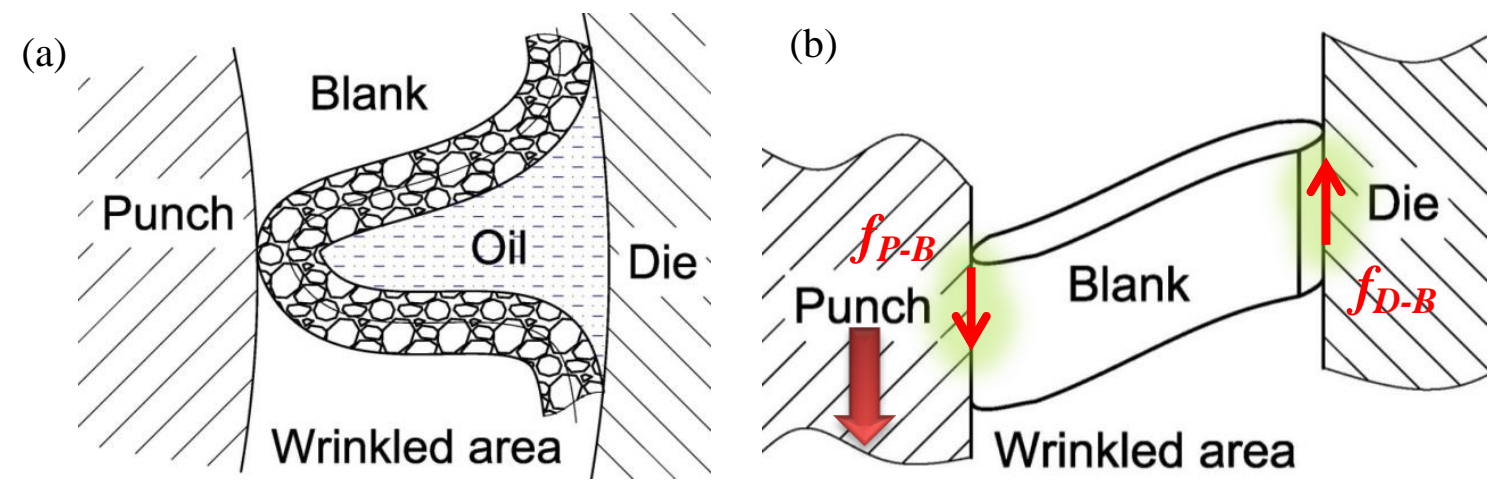

Figure 8 Illustration of local friction among the wrinkled blank, the punch and the die from (a) top view and (b) sectional view

\section{Finite element sSimulation results}

According to the geometry of the MHDD system, a micro scale finite element simulation model was built up. The blank was modelled with shell elements, governed by the fully integration formula and followed the 3-Parameter Barlat material model. Regarding the size effects, the Voronoi structure was utilised, where the Voronoi cells had similar size and shape to the grains on the annealed SUS304 sheets. Further, all the cells were classified into five groups with individual material properties, and they were obtained from the aforementioned tensile tests in Section 2.1. As to the friction change in the micro scale, the open and closed lubricant pockets theory was adopted in this model. Correspondingly, cells with positions outside a critical radius were defined as the open lubricant pocket area. Additionally, the open lubricant pocket area was assigned a small friction coefficient, while the other cells were of closed lubricant pocket area and had high friction coefficient. Furthermore, the area of the open lubricant pocket zone was increased with the hydraulic pressure. Fig. 9 illustrated the Voronoi blank models with open and closed lubricant pocket areas. Therefore, although constant friction coefficients were used in the simulation model and the hydraulic pressure was treated as a constant load on the blank, the 
friction change in the MHDD can be presented. One quarter of the MHDD system was modelled to accelerate computational speed. After simulation, coordinates of the nodes on the drawn cup's top mouth were exported for the wrinkling calculation. Similarly, the diameter difference between the maximum and minimum radii of the nodes was utilised as the wrinkling judgement. However, as shell elements were used, the inner and outer surfaces of the drawn cup cannot be considered separately and only the coordinates of the nodes were used. Fig. 10 shows the wrinkling from simulation results.

(a)

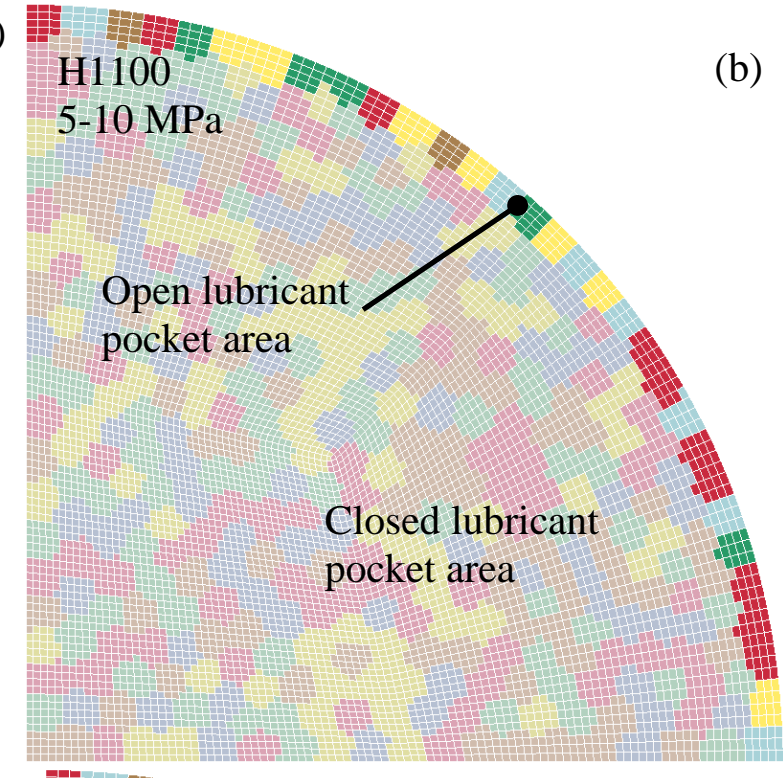

(c)

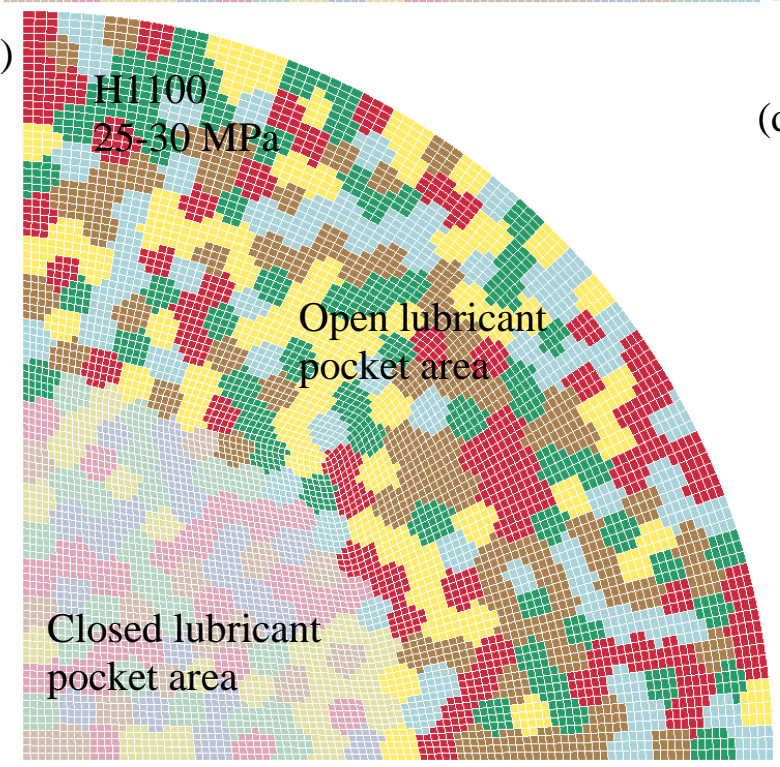

(b)

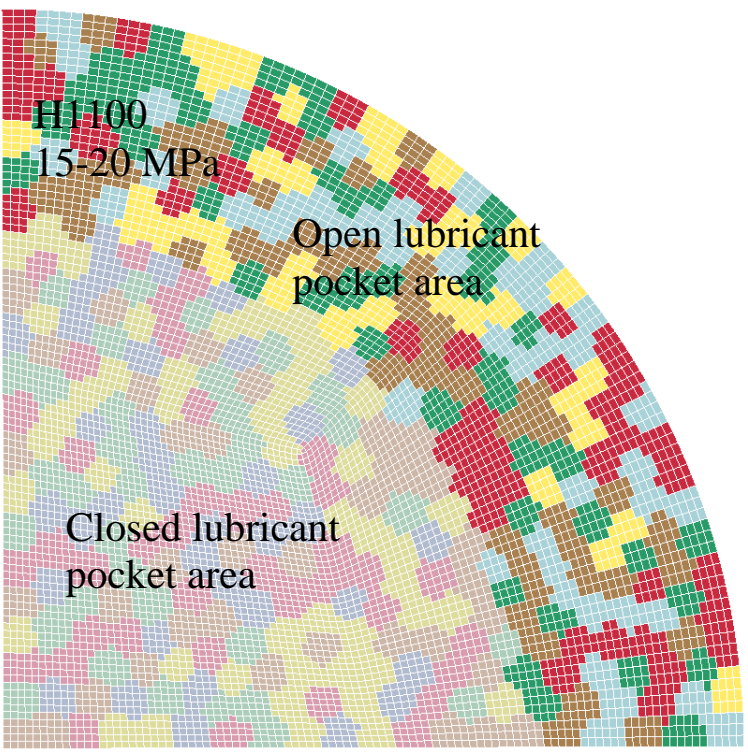

(d)

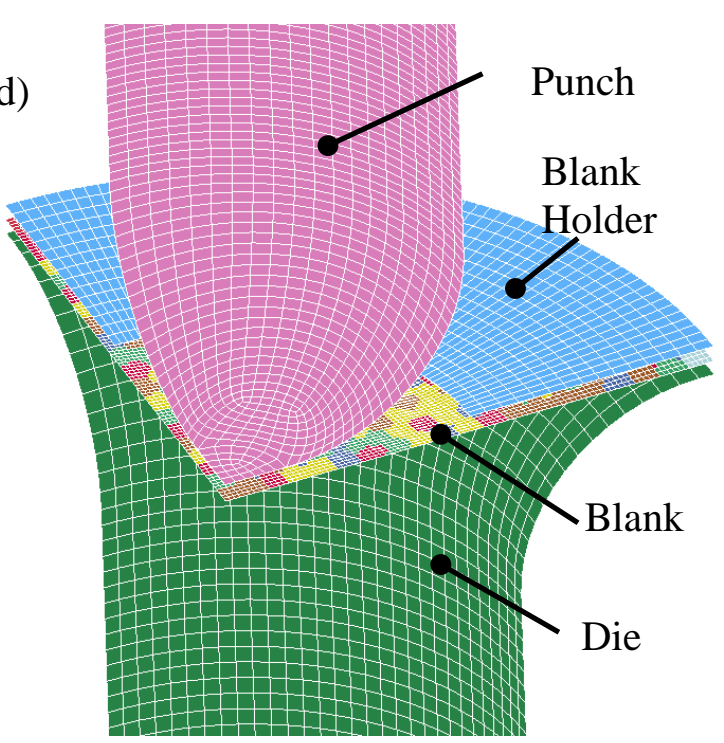

Figure 9 (a-c) Voronoi blank models under different hydraulic pressure where the bright areas are the open lubricant pocket areas and the transparent areas are the closed lubricant pocket areas, and (b) the whole MHDD model 


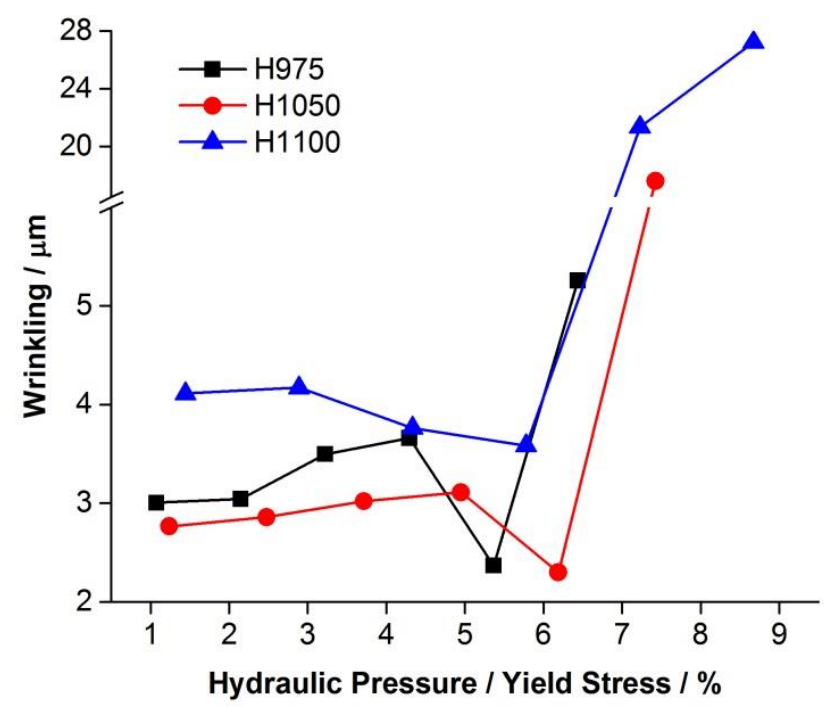

Figure 10 Wrinkling from the simulation results

The general wrinkling development in simulation was the same as that in the MHDD experiments. The wrinkling values for each blank group increased to a local peak and then dropt to a local minimum. Thereafter, the wrinkling increased till the maximum experimental pressure. Moreover, the critical hydraulic pressure range was between three and six percent of the blank's initial yield stress. Additionally, all nodes were at middle thickness of the elements and the thickness was ignored in wrinkling calculation. Therefore, the wrinkling values in simulation were different from that in the experiments. The general wrinkling trend and the critical pressure range were well predicted in the simulation and matched the experimental data. This proved that the size effects and friction change in the MHDD are key factors affecting drawn cups in the MHDD.

\section{Conclusions}

The hydraulic pressure influences the quality of the drawn cups regarding the wrinkling and earing at the cup mouth area. There is a critical hydraulic pressure range, under which the wrinkling and earing developing trends change and reach local extremum, for each material group. Moreover, the wrinkling and earing developing trends also change twice in the critical pressure range. Furthermore, the critical hydraulic pressure is about three to six percent of the blank's initial yield stress.

When the pressure is less than one tenth of material's initial yield stress, effects of the hydraulic pressure are mainly presented by the change in friction and shape of the blank during the micro hydro deep drawing process. The earing is strongly related to the wrinkling with a reverse developing tendency. The friction plays a significant role in the micro hydro deep drawing and greatly impacts the wrinkling and earing.

Influences of the size effects and friction change on the drawn cups are proved by the simulation. 
The Voronoi simulation model with separated consideration of the open and closed lubricant pocket areas can reveal the same wrinkling development trend as that in the experiments. Moreover, the predicted critical hydraulic pressure range was the same as that in the experiments.

\section{Acknowledgements}

The first author greatly thanks for the financial support from the Chinese Scholarship Council (CSC201206160011) and the International postgraduate tuition award (IPTA) offered by the University of Wollongong. The authors thank for the help in hydraulic system from Prof. Xiaofeng He from Huazhong University of Science and Technology. The authors also thank Mr. Xiaoguang Ma and Mr. Fanghui Jia for their assistant in the experiments.

\section{References}

[1] A.K. Sharma, D.K. Rout, Finite element analysis of sheet Hydromechanical forming of circular cup, J Mater Process Technol, 209 (2009) 1445-1453.

[2] S.H. Zhang, J. Danckert, Development of hydro-mechanical deep drawing, J Mater Process Technol, 83 (1998) 14-25.

[3] R. Zhang, L. Lang, R. Zafar, L. Lin, W. Zhang, Investigation into thinning and spring back of multilayer metal forming using hydro-mechanical deep drawing (HMDD) for lightweight parts, Int J Adv Manuf Tech, 82 (2015) 817-826.

[4] S. Bagherzadeh, M.J. Mirnia, B. Mollaei Dariani, Numerical and experimental investigations of hydro-mechanical deep drawing process of laminated aluminum/steel sheets, J Manuf Process, 18 (2015) 131-140.

[5] M. Janbakhsh, M. Riahi, F. Djavanroodi, A Practical Approach to Analysis of Hydromechanical Deep Drawing of Superalloy Sheet Metals by Using Finite Element Method, Int J Adv Manuf Technol, 6 (2013) 1-7.

[6] H. Wang, L. Gao, M. Chen, Hydrodynamic deep drawing process assisted by radial pressure with inward flowing liquid, Int J Mech Sci, 53 (2011) 793-799.

[7] B. Chen, Y. Xu, S. Yuan, Investigation into influence of pre-bulging on subsequent hydrodynamic deep drawing, Rev. Adv. Mater. Sci, 33 (2013) 423-428.

[8] H. Sato, K. Manabe, D.B. Wei, Z.Y. Jiang, D. Kondo, Micro Sheet Hydroforming Process of Ultra-Thin Pure Titanium Foil, in: Key Eng. Mater., Trans Tech Publ, 2015, pp. 397-401.

[9] I. Irthiea, G. Green, S. Hashim, A. Kriama, Experimental and numerical investigation on micro deep drawing process of stainless steel 304 foil using flexible tools, Int. J. Mach. Tools Manuf, 76 (2014) 21-33.

[10] F. Vollertsen, Effects on the deep drawing diagram in micro forming, Prod. Eng. Res. Devel., 6 (2012) 11-18.

[11] A. Molotnikov, R. Lapovok, C.F. Gu, C.H.J. Davies, Y. Estrin, Size effects in micro cup drawing, Mater Sci Eng A-struct, 550 (2012) 312-319.

[12] H. Sato, K.-i. Manabe, D. Wei, Z. Jiang, S. Alexandrov, Tribological behavior in microsheet hydroforming, Tribol Int, 97 (2016) 302-312.

[13] T. Shimizu, M. Ogawa, M. Yang, K.-i. Manabe, Plastic anisotropy of ultra-thin rolled phosphor bronze foils and its thickness strain evolution in micro-deep drawing, Mater Design, 56 
(2014) 604-612.

[14] J. Xu, X. Zhu, D. Shan, B. Guo, T.G. Langdon, Effect of grain size and specimen dimensions on micro-forming of high purity aluminum, Mater Sci Eng A-struct, 646 (2015) 207217.

[15] Z. Xu, L. Peng, X. Lai, M. Fu, Geometry and grain size effects on the forming limit of sheet metals in micro-scaled plastic deformation, Mater Sci Eng A-struct, 611 (2014) 345-353.

[16] M. Fu, W. Chan, A review on the state-of-the-art microforming technologies, Int J Adv Manuf Tech, 67 (2013) 2411-2437.

[17] C. Wang, B. Guo, D. Shan, M. Zhang, X. Bai, Tribological behaviors in microforming considering microscopically trapped lubricant at contact interface, Int J Adv Manuf Tech, 71 (2014) 2083-2090.

[18] C.M. Ho, Y.C. Tai, Micro-electro-mechanical-systems (MEMS) and fluid flows, Annu. Rev. Fluid Mech., 30 (1998) 579-612.

[19] M. Abbasi, M. Ketabchi, T. Labudde, U. Prahl, W. Bleck, New attempt to wrinkling behavior analysis of tailor welded blanks during the deep drawing process, Mater Design, 40 (2012) 407-414.

[20] J. Raja, B. Muralikrishnan, S. Fu, Recent advances in separation of roughness, waviness and form, Precis Eng, 26 (2002) 222-235. 